\title{
How Could We Prevent Spread of the Coronavirus without Deteriorating Economy?
}

\author{
Yasunori Fujita \\ Keio University, Tokyo, Japan \\ Email:yfujita@econ.keio.ac.jp
}

How to cite this paper: Fujita, Y. (2020). How Could We Prevent Spread of the Coronavirus without Deteriorating Economy? Modern Economy, 11, 1280-1287. https://doi.org/10.4236/me.2020.117091

Received: April 19, 2020

Accepted: July 12, 2020

Published: July 15, 2020

Copyright $\odot 2020$ by author(s) and Scientific Research Publishing Inc. This work is licensed under the Creative Commons Attribution International License (CC BY 4.0).

http://creativecommons.org/licenses/by/4.0/ (c) (i) Open Access

\begin{abstract}
In the present paper, we attempt to find a way of preventing the spread of the new coronavirus without deteriorating the economy, by constructing an intertemporal model based on the dynamic optimization theory. More precisely, following the procedures used in dynamic optimization, we derive the optimal intertemporal path of the contact rate among the workers in an economy where the new coronavirus is spreading widely. It is revealed that under the optimal regulation, the workers should contact with other workers less and less at first, and then contact with other workers more and more until the contact rate reaches certain point.
\end{abstract}

Keywords

Coronavirus, Dynamic Optimization, Hamiltonian, Euler Equation, Contact Rate

\section{Introduction}

Since the outbreak of the new coronavirus, many attempts have been made to reveal empirically its impacts on various economic aspects such as consumption, stock market, uncertainty and so on (Barro, Ursua, \& Weng, 2020; Baker, Bloom, Davis, Kost, Sammon, \& Viratyosin, 2020; Baker, Farrokhnia, Meyer, Pagel, \& Yannelis, 2020; Baker, Bloom, Davis, \& Terry, 2020; Watanabe, 2020; and so on). In the present paper, based on these data analyses, we attempt to build the foundation of theoretical analysis of the new coronavirus problem by making use of the dynamic optimization theory whose masterpieces include Seierstad and Sydsaeter (1986), Kamien and Schwartz (1991) and Chiang (1992). The question we pose is how we can prevent the spread of the new coronavirus without deteriorating the economy, by controlling the contact rate among workers. We follow the standard procedures in the dynamic optimization theory 
and reveal that the workers should contact with other workers less and less at first, and then contact with other workers more and more until the contact rate reaches certain point. The present paper is different from Fujita (2020a, 2020b), which also attempted to find a way of controlling the spread of the coronavirus without deteriorating the economy, in that Fujita (2020a, 2020b) examined the optimal timing of the lockdown based on the optimal stopping theory; the present paper focuses on the continuous control of the spread of coronavirus based on the dynamic optimization theory.

Structure of this paper is as follows. Section 2 lays out the basic model of dynamic optimization and Section 3 derives the optimal intertemporal path of the contact rate among the workers. Concluding comments are made in Section 4.

\section{Basic Model}

Let us consider an intertemporal economy that consists of one firm and $N$ workers, of whom $n(t)$ workers are infected with the coronavirus but have not presented symptoms yet. We assume that time passes continuously and the time horizon is infinite. In period $t$ the $n(t)$ infected-but-no-symptom workers transmit the virus to $\alpha(t) n(t)$ workers, of whom a fraction of $\left(1-\frac{n(t)}{N}\right)$ is newly infected, and the government controls the contact rate $\alpha(t)$ at costs of $C(\alpha(t))$ where $C(0)=\infty, \frac{\mathrm{d} C}{\mathrm{~d} \alpha(t)}<0$ and $\frac{\mathrm{d}^{2} C}{\mathrm{~d} \alpha(t)^{2}}>0$, which means that more costs are necessary to keep the contact rate lower. Throughout the present paper, for the sake of simplicity of the analysis, we specify $C(\alpha(t))$ as $\frac{c}{\alpha(t)}$, by letting $c$ be a positive constant.

In each period, the infected-but-no-symptom workers are assumed to present symptoms at a constant rate $e$ and exit from the group of the infected-but-nosymptom workers. So that, the law of motion of $n(t)$ is expressed as

$$
\frac{\mathrm{d} n}{\mathrm{~d} t}=-e n(t)+n(t) \alpha(t)\left(1-\frac{n(t)}{N}\right)
$$

As for the amount of GDP, as in Fujita (2020a, 2020b), we assume it decreases as the number of workers who present symptoms en $(t)$ increases. In order to simplify the analysis, letting $Y$ denote the amount of GDP when no worker presents symptoms, we specify the amount of GDP in period $t$ as $Y-\theta e n(t)$, where $\theta$ is a positive constant.

Letting $r$ denote discount rate, and assuming the government to maximize discounted values of sum of the GDP minus the costs $C(\alpha(t))$, the optimal problem for the government reduces to a typical one as in Section 9 of Kamien and Schwartz (1991):

$$
\max \int_{0}^{\infty} \mathrm{e}^{-r t}\left\{Y-\theta e n(t)-\frac{c}{\alpha(t)}\right\} \mathrm{d} t
$$




$$
\text { Subject to } \frac{\mathrm{d} n}{\mathrm{~d} t}=-b e(t)+n(t) \alpha(t)\left(1-\frac{n(t)}{N}\right)
$$

Following the standard procedure for solution, by letting $m(t)$ a multiplier, we define the current value Hamiltonian equation as follows:

$$
H=Y-\theta e n(t)-\frac{c}{\alpha(t)}+m(t)\left\{-e n(t)+n(t) \alpha(t)\left(1-\frac{n(t)}{N}\right)\right\}
$$

Since the government solves the optimal problem with control variable and state variable being $\alpha(t)$ and $n(t)$ respectively, we obtain the first order condition $\frac{\mathrm{d} H}{\mathrm{~d} \alpha}=0$ and the Euler equation $\frac{\mathrm{d} m}{\mathrm{~d} t}=r m(t)-\frac{\mathrm{d} H}{\mathrm{~d} n}$, respectively, as

$$
\begin{gathered}
m(t)=\frac{-C}{\alpha(t)^{2} n(t)\left(1-\frac{n(t)}{N}\right)} ; \\
\frac{\mathrm{d} m}{\mathrm{~d} t}=(r+e-\alpha(t)) m+\frac{2 m(t) n(t) \alpha(t)}{N}+\theta e .
\end{gathered}
$$

The transversality condition is satisfied since the model in the present paper has infinite time horizon.

\section{Derivation of the 0ptimal Intertemporal Path}

By making use of (5) and differentiating $\alpha(t)$ with respect to $t, \frac{\mathrm{d} \alpha}{\mathrm{d} t}=\frac{\mathrm{d} \alpha}{\mathrm{d} m} \frac{\mathrm{d} m}{\mathrm{~d} t}$, we can eliminate $m(t)$ in (6), to get the system of motions of $n(t)$ and $\alpha(t)$ as (1) and

$$
\frac{\mathrm{d} \alpha}{\mathrm{d} t}=\alpha(t)\left\{-\frac{1}{2}(r+e) c+\left(\frac{1}{2}-\frac{n(t)}{N}\right) c \alpha(t)+\frac{\theta e}{2}\left(1-\frac{n(t)}{N}\right) n(t) \alpha(t)^{2}\right\} .
$$

Thus, we have the loci of $\frac{\mathrm{d} n}{\mathrm{~d} t}=0$ and $\frac{\mathrm{d} \alpha}{\mathrm{d} t}=0$ as

$$
\begin{gathered}
\alpha(t)=\frac{b}{1-\frac{n(t)}{N}} ; \\
\alpha(t)=\frac{1}{2\left(1-\frac{n}{N}\right) n}\left\{\left(\frac{2 n}{N}-1\right) c+\sqrt{\left(\frac{2 n}{N}-1\right)^{2} c^{2}+4 \theta e n\left(1-\frac{n}{N}\right)(r+e) c} .\right.
\end{gathered}
$$

In order to sketch the direction of the movement compatible with these two equations, let us first consider the $\frac{\mathrm{d} n}{\mathrm{~d} t}=0$ locus (Equation (8)), which is drawn as an upward sloping curve in $n$ - $\alpha$ space. Since the $\frac{\mathrm{d} n}{\mathrm{~d} t}=0$ locus contains all points in the plane where $\frac{\mathrm{d} n}{\mathrm{~d} t}$ is zero, points that are not on the $\frac{\mathrm{d} n}{\mathrm{~d} t}=0$ locus have nonzero derivatives. Since (8) is continuous in $n$ and $\alpha$, regions of positive and negative derivatives must be separated by the $\frac{\mathrm{d} n}{\mathrm{~d} t}=0$ locus. Since the right 
hand side of (1) is increasing function with respect to $\alpha$, we have $\frac{\mathrm{d} n}{\mathrm{~d} t}>0$ above the $\frac{\mathrm{d} n}{\mathrm{~d} t}=0$ locus, while $\frac{\mathrm{d} n}{\mathrm{~d} t}<0$ below the $\frac{\mathrm{d} n}{\mathrm{~d} t}=0$ locus.

Similarly, let us consider the $\frac{\mathrm{d} \alpha}{\mathrm{d} t}=0$ locus (Equation (9)), which is drawn as a U-shaped curve in $n$ - $\alpha$ space. Since the $\frac{\mathrm{d} \alpha}{\mathrm{d} t}=0$ locus contains all points in the plane where $\frac{\mathrm{d} \alpha}{\mathrm{d} t}$ is zero, points that are not on the $\frac{\mathrm{d} \alpha}{\mathrm{d} t}=0$ locus have nonzero derivatives. Since (9) is continuous in $n$ and $\alpha$, regions of positive and negative derivatives must be separated by the $\frac{\mathrm{d} \alpha}{\mathrm{d} t}=0$ locus. Since the right hand side of (7) is increasing function with respect to $\alpha$, we have $\frac{\mathrm{d} \alpha}{\mathrm{d} t}>0$ above the $\frac{\mathrm{d} \alpha}{\mathrm{d} t}=0$ locus, while $\frac{\mathrm{d} \alpha}{\mathrm{d} t}<0$ below the $\frac{\mathrm{d} \alpha}{\mathrm{d} t}=0$ locus.

Since the equilibria are determined as the intersections of the loci of $\frac{\mathrm{d} n}{\mathrm{~d} t}=0$ and $\frac{\mathrm{d} \alpha}{\mathrm{d} t}=0$, we have two equilibria in Figure 1. To characterize each equilibrium, we linearize the system of (8) and (9) in the neighborhood of the equilibrium as

$$
\frac{\mathrm{d} n}{\mathrm{~d} t}=\left\{-e+\alpha(t)\left(1-\frac{2 n(t)}{N}\right)\right\} \mathrm{d} n+n(t)\left(1-\frac{n(t)}{N}\right) \mathrm{d} \alpha
$$

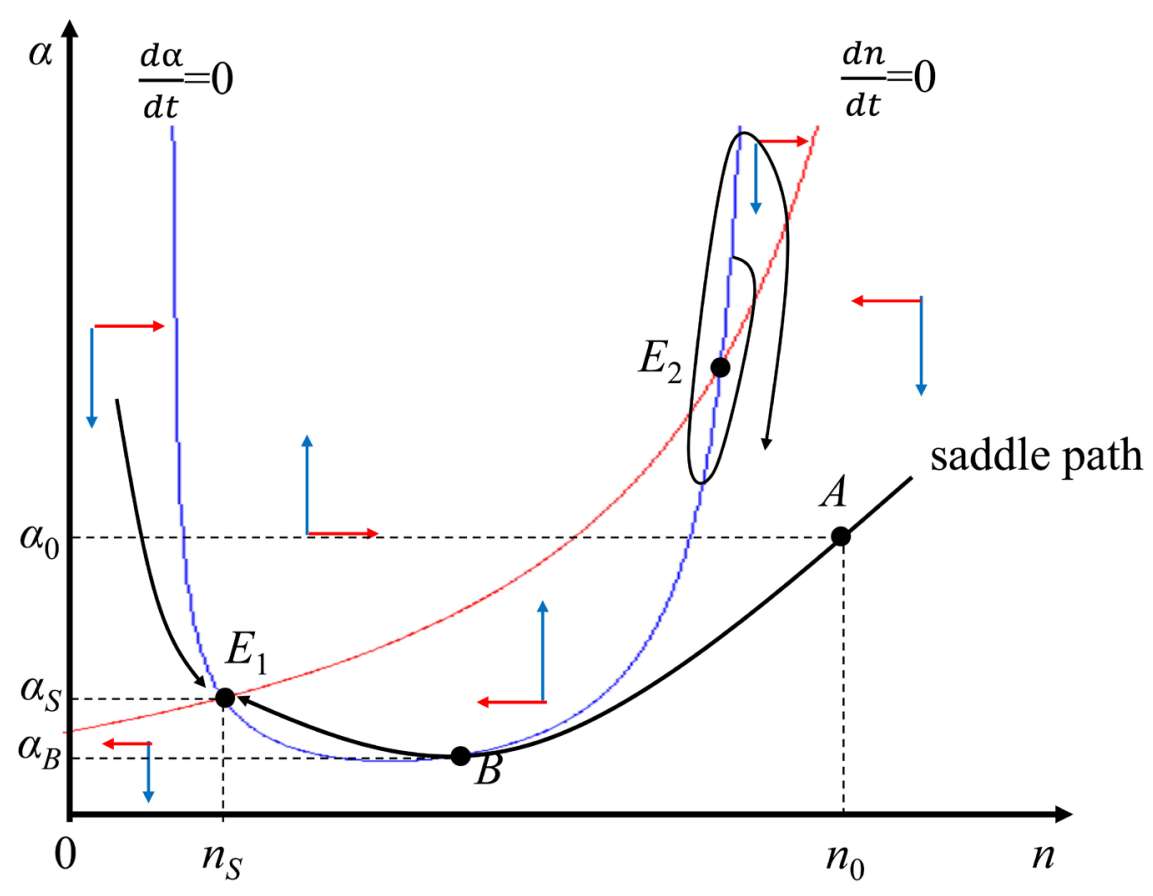

Figure 1. Phase diagram and saddle path. 


$$
\begin{aligned}
\frac{\mathrm{d} \alpha}{\mathrm{d} t}= & \alpha(t)\left\{-\frac{c}{N}+\frac{\theta e}{2}\left(1-\frac{2 n(t)}{N}\right) \alpha(t)\right\} \mathrm{d} n \\
& +\left\{-\frac{1}{2}(r+e)+2\left(\frac{1}{2}-\frac{n(t)}{N}\right) c \alpha(t)+\frac{3 \theta e}{2}\left(1-\frac{n(t)}{N}\right) n(t) \alpha(t)^{2}\right\} \mathrm{d} \alpha .
\end{aligned}
$$

Letting $a_{11}, a_{12}, a_{21}$ and $a_{22}$ denote $-e+\alpha(t)\left(1-\frac{2 n(t)}{N}\right), n(t)\left(1-\frac{n(t)}{N}\right)$, $\alpha(t)\left\{-\frac{c}{N}+\frac{\theta e}{2}\left(1-\frac{2 n(t)}{N}\right) \alpha(t)\right\}$ and

$\left\{-\frac{1}{2}(r+e)+2\left(\frac{1}{2}-\frac{n(t)}{N}\right) c \alpha(t)+\frac{3 \theta e}{2}\left(1-\frac{n(t)}{N}\right) n(t) \alpha(t)^{2}\right\}$ respectively, $\frac{\mathrm{d} n}{\mathrm{~d} t}$ and $\frac{\mathrm{d} \alpha}{\mathrm{d} t}$ are expressed as

$$
\begin{gathered}
\frac{\mathrm{d} n}{\mathrm{~d} t}=a_{11} \mathrm{~d} n+a_{12} \mathrm{~d} \alpha ; \\
\frac{\mathrm{d} \alpha}{\mathrm{d} t}=a_{21} \mathrm{~d} n+a_{22} \mathrm{~d} \alpha .
\end{gathered}
$$

The linearized $\frac{\mathrm{d} n}{\mathrm{~d} t}=0$ locus has slope $\frac{\mathrm{d} \alpha}{\mathrm{d} n}=-\frac{a_{11}}{a_{12}}$ while the linearized $\frac{\mathrm{d} \alpha}{\mathrm{d} t}=0$ locus has slope $\frac{\mathrm{d} \alpha}{\mathrm{d} n}=-\frac{a_{21}}{a_{22}}$. It is known that if $a_{11} a_{22}-a_{12} a_{21}<0$, the intersection is saddle point; if $a_{11} a_{22}-a_{12} a_{21}>0$, the intersection is unstable point. Thus, it follows that $a_{11} a_{22}-a_{12} a_{21}<0$ holds if and only if $-\frac{a_{11}}{a_{12}}>-\frac{a_{21}}{a_{22}}$; that is, the linearized $\frac{\mathrm{d} n}{\mathrm{~d} t}=0$ locus is steeper than the linearized $\frac{\mathrm{d} \alpha}{\mathrm{d} t}=0$ locus. On the other hand, $a_{11} a_{22}-a_{12} a_{21}>0$ holds if and only if $-\frac{a_{11}}{a_{12}}<-\frac{a_{21}}{a_{22}}$; that is, the linearized $\frac{\mathrm{d} n}{\mathrm{~d} t}=0$ locus is less steep than the linearized $\frac{\mathrm{d} \alpha}{\mathrm{d} t}=0$ locus. Therefore, we see that point $E_{1}$ is a saddle point and $E_{2}$ is an unstable point in Figure 1.

The solution to the optimal problem (2) is summarized by the saddle path in Figure 1, which means, the government should change $n$ and $\alpha$ along the saddle path. If we assume that the initial amount of $n$ is $n_{0}$, we see that the government should reduce $n(t)$ monotonically until it reaches the steady state value $n_{S}$ (point $E_{1}$ ); the government should first let $\alpha(t)$ jump to $\alpha_{0}$ (point $A$ ), reduce it until it reaches $\alpha_{B}$ (point $B$ ) and increase it until it reaches the steady state value $\alpha_{S}$ (point $E_{1}$ ). By focusing on the values of $\alpha$, we can derive the following proposition 1.

Proposition 1:

In the situation where initial number of infected works is sufficiently large, under the optimal regulation, workers should contact with other 
workers less and less at first, and then contact with other workers more and more until the contact rate reaches certain point.

We see from (7) and (8), if $c$ or $\theta$ increases, the saddle path moves upwards (from DD curve to D'D' curve) since the $\frac{\mathrm{d} \alpha}{\mathrm{d} t}=0$ locus upward, keeping the $\frac{\mathrm{d} n}{\mathrm{~d} t}=0$ locus unchanged as in Figure 2; if $e$ increases, the saddle path moves upwards since both the $\frac{\mathrm{d} \alpha}{\mathrm{d} t}=0$ locus and the $\frac{\mathrm{d} n}{\mathrm{~d} t}=0$ locus shift upwards as in Figure 3 (from DD curve to D'D' curve). Since $c$ and $\theta$ are parameters of the

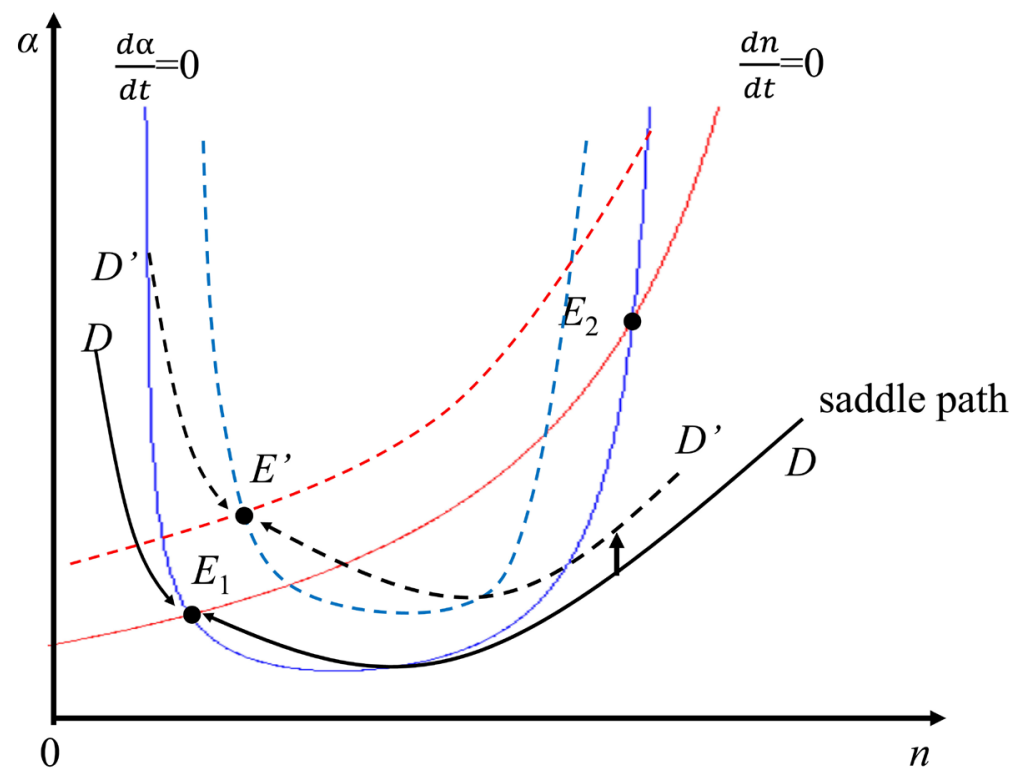

Figure 2. Effect of increase in $c$ or $\theta$ on the saddle path.

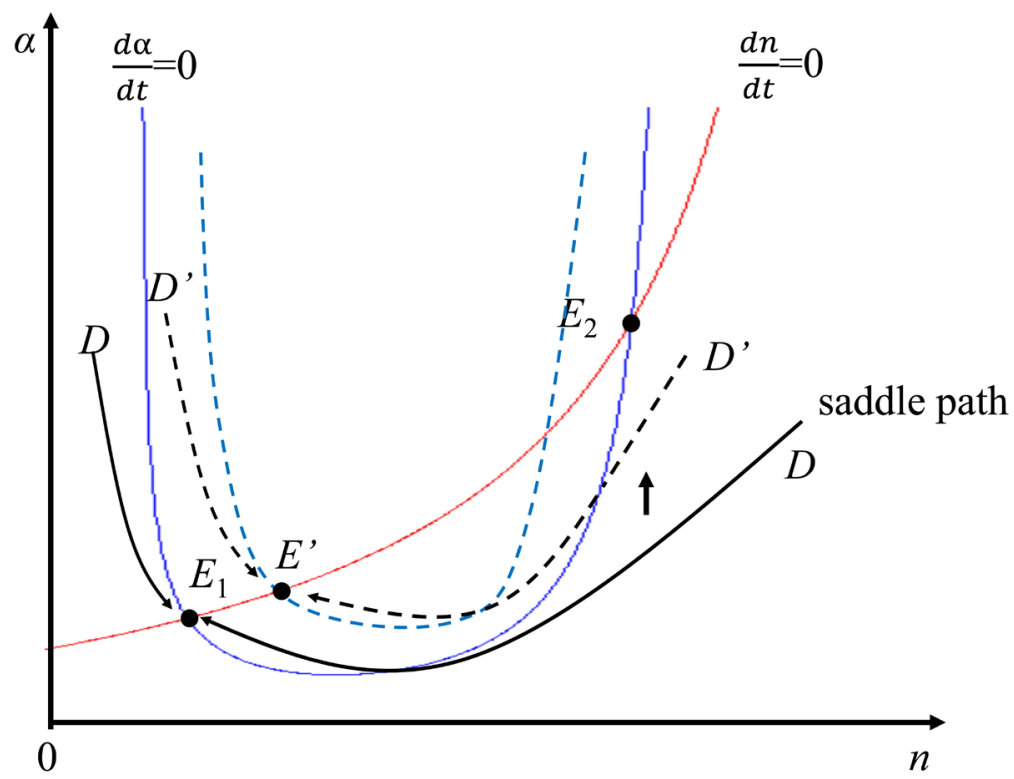

Figure 3. Effect of increase in $e$ on the saddle path. 
cost of the coronavirus, while $e$ is a parameter that represents the rate of those who present symptoms, we can derive the following proposition 2 by focusing on the values of $\alpha$.

Proposition 2:

Government should regulate the workers so that their contact rate is higher than before if the cost of the coronavirus or the rate of those who present symptoms gets higher.

\section{Conclusion}

In the present paper, we attempted to build the foundation of theoretical analysis of the new coronavirus problem by making use of the dynamic optimization theory. The question we posed was how we can prevent the spread of the new coronavirus without deteriorating the economy, by controlling the contact rate among the workers. It was revealed that the workers should contact with other workers less and less at first, and then contact with other workers more and more until the contact rate reaches certain point.

Many researchers have been attempting to solve the coronavirus problems. We truly hope this research note will contribute to constructing better theoretical frameworks to examine how to control the spread of the coronavirus.

\section{Conflicts of Interest}

The author declares no conflicts of interest regarding the publication of this paper.

\section{References}

Baker, S. R., Bloom, N., Davis, S. J., \& Terry, S. J. (2020). COVID-Induced Economic Uncertainty. NBER Working Paper Series 26983. https://doi.org/10.3386/w26983

Baker, S. R., Bloom, N., Davis, S. J., Kost, K. J., Sammon, M. C., \& Viratyosin, T. (2020). The Unprecedented Stock Market Impact of Covid-19. NBER Working Paper Series 26945. https://doi.org/10.3386/w26945

Baker, S. R., Farrokhnia, R. A., Meyer, S., Pagel, M., \& Yannelis, C. (2020). How Does Household Spending Respond to an Epidemic? Consumption during the 2020 Covid-19 Pandemic. NBER Working Paper Series 26949. https://doi.org/10.3386/w26949

Barro, R., Ursua, J. F., \& Weng, J. (2020). The Coronavirus and the Great Influenza Epidemic: Lessons from the "Spanish Flu" for the Coronavirus's Potential Effects on Mortality and Economic Activity. NBER Working Paper Series 26866.

https://doi.org/10.3386/w26866

Chiang, A. C. (1992). Elements of Dynamic Optimization. New York: McGraw-Hill.

Fujita, Y. (2020a). When Should We Start the Lockdown and How Long Should It Be? Modern Economy, 11, 1007-1011. https://doi.org/10.4236/me.2020.115075

Fujita, Y. (2020b). How Much Should Government Compensate Firms for Suspension of Their Businesses in Order to Fight off the New Coronavirus? Theoretical Economics Letters, 10, 600-606. https://doi.org/10.4236/tel.2020.103038

Kamien, M. I., \& Schwartz, N. L. (1991). Dynamic Optimization: The Calculus of Variations and Optimal Control in Economics and Management. Amsterdam: North-Holland. 
Seierstad, A., \& Sydsaeter, K. (1986). Optimal Control Theory with Economic Applications. Amsterdam: Elsevier.

Watanabe, T. (2020). The Responses of Consumption and Prices in Japan to the COVID-19 Crisis and the Tohoku Earthquake. Working Paper Series, CARF-F-476. 